\title{
Quantitative Diagnosis of Rotor Vibration Fault Using Process Power Spectrum Entropy and Support Vector Machine Method
}

\author{
Cheng-Wei Fei, ${ }^{1}$ Guang-Chen Bai, ${ }^{1}$ Wen-Zhong Tang, ${ }^{2}$ and Shuang $\mathrm{Ma}^{3}$ \\ ${ }^{1}$ School of Energy and Power Engineering, Beijing University of Aeronautics and Astronautics, Beijing 100191, China \\ ${ }^{2}$ School of Computer Science and Engineering, Beijing University of Aeronautics and Astronautics, Beijing 100191, China \\ ${ }^{3}$ School of Life Science, Beijing Normal University, Beijing 100875, China \\ Correspondence should be addressed to Cheng-Wei Fei; feicw544@163.com
}

Received 19 November 2013; Revised 8 March 2014; Accepted 10 March 2014; Published 31 March 2014

Academic Editor: Valder Steffen

Copyright (C) 2014 Cheng-Wei Fei et al. This is an open access article distributed under the Creative Commons Attribution License, which permits unrestricted use, distribution, and reproduction in any medium, provided the original work is properly cited.

\begin{abstract}
To improve the diagnosis capacity of rotor vibration fault in stochastic process, an effective fault diagnosis method (named Process Power Spectrum Entropy (PPSE) and Support Vector Machine (SVM) (PPSE-SVM, for short) method) was proposed. The fault diagnosis model of PPSE-SVM was established by fusing PPSE method and SVM theory. Based on the simulation experiment of rotor vibration fault, process data for four typical vibration faults (rotor imbalance, shaft misalignment, rotor-stator rubbing, and pedestal looseness) were collected under multipoint (multiple channels) and multispeed. By using PPSE method, the PPSE values of these data were extracted as fault feature vectors to establish the SVM model of rotor vibration fault diagnosis. From rotor vibration fault diagnosis, the results demonstrate that the proposed method possesses high precision, good learning ability, good generalization ability, and strong fault-tolerant ability (robustness) in four aspects of distinguishing fault types, fault severity, fault location, and noise immunity of rotor stochastic vibration. This paper presents a novel method (PPSE-SVM) for rotor vibration fault diagnosis and real-time vibration monitoring. The presented effort is promising to improve the fault diagnosis precision of rotating machinery like gas turbine.
\end{abstract}

\section{Introduction}

Vibration is a momentous fault source of rotating machinery like an aeroengine and seriously impacts on the security and reliability of machine system operation [1]. With the development of the high performance and high reliability of rotating machinery, vibration fault needs to be predicted and inhibited early [1-3]. Therefore, how to predict and control rotor vibration faults is one of hot issues in preventing the failures of mechanical system, which leads to the advance of feasible and effective fault diagnosis methods [3-8]. However, most of present vibration analysis techniques need a mass of vibration samples to establish a fault diagnosis model to diagnose the vibration faults from a qualitative perspective. In fact, these fault analysis methods possess some blindness in fault diagnosis, which seriously influences diagnosis accuracy, due to being short of describing diagnosis results from a process and quantitative perspective. Meanwhile, it is always difficult to gain large number of vibration fault data. In order to improve the validity of fault diagnosis, the process and quantitative factors should be considered to ascertain fault types, failure severity, fault location, and even development tendency. The development of information entropy theory is promising to quantitatively analyze rotor vibration conditions and describe the uncertainty degree of vibration signal $[6,9,10]$. Information entropy technique as a process fault diagnosis method has been widely researched and applied to machinery fault diagnosis $[8,10-12]$. Specially, for quantitative diagnosis of rotor vibration faults, process information entropy method also called as Process Power Spectrum Entropy (PPSE) method was proposed and proved to be effective [11]. Although these techniques are feasible in diagnosing some simple faults, due to the complexity of vibration signals for large rotating machinery, information fusion technology is required to synthetically deal with multisensor information in order to ameliorate fault analysis precision $[3,4,13,14]$. Support Vector Machine (SVM) is a new intelligent pattern recognition method, which uses structural 
risk minimization instead of empirical risk minimization to solve small-sample, nonlinear, high-dimensional problems, and so on. SVM has been proved to embody various strengths of complete theory, good adaptability, global optimization, short training time, and good generalization ability in fault diagnosis $[3,8,14-18]$.

The purpose of this present study attempts to propose an effective approach which is Process Power Spectrum Entropy-SVM (PPSE-SVM) method for rotor vibration fault diagnosis based on PPSE method and SVM theory. This method is promising to resolve the randomization of vibration fault and the difficulty of extracting vibration fault samples. Based on rotor vibration simulation workbench, four typical faults are simulated and their vibration data are gained. The PPSE values (i.e., information features) of these data are calculated based on PPSE method to construct eigenvectors as fault diagnosis samples. SVM fault diagnosis model was established based on the fault diagnosis samples. The feasibility and validity of PPSE-SVM method are verified by diagnosing rotor vibration fault types, failure severity, fault point, and noise immunity.

\section{Process Power Spectrum Entropy Method}

Information entropy was first propounded by Shannon to evaluate information capacity [3,6-12]. Assuming that $\mathbf{M}$ is a Lebesgue space with an algebra $\delta$ generated by a measurable set $H$ and a measure $\mu(\mu(\mathbf{M})=1)$ and the space $\mathbf{M}$ may be decomposed as a limited partitioning $\mathbf{A}=\left(A_{i}\right)$ which is an incompatible set satisfying $\mathbf{M}=\bigcup_{i=1}^{n} A_{i}$ and $A_{i} \cap A_{j}=0, \forall i \neq j$, based on information entropy theory, the information entropy $E(\mathbf{A})$ of $\mathbf{A}$ is denoted as [3]

$$
E(\mathbf{A})=-\sum_{i=1}^{n} \mu\left(A_{i}\right) \log \mu\left(A_{i}\right),
$$

where $\mu\left(A_{i}\right)$ is the measurement of sample $A_{i}, i=1,2, \ldots, n$.

As shown in (1), information entropy $E$ shows actually the chaotic degree of uncertain factors in a system. More disorder and randomness of the system always lead to that the corresponding information entropy values become greater, and vice versa [7].

The Power Spectrum Entropy (PSE) values of rotor vibration signal can be extracted from the features of vibration signals in frequency domain from an energy perspective when $X(\omega)$ is the discrete Fourier transform of a singlechannel signal $\left\{x_{t}\right\}$ as follows:

$$
X(\omega)=\frac{1}{2 n \pi} \sum_{t=1}^{N-1} x_{t} e^{-j \omega t} .
$$

Thus, the power spectrum of $X(\omega)$ is defined by

$$
S(\omega)=\frac{1}{2 \pi N}|X(\omega)|^{2}
$$

due to the energy conserve law in the signal transformation process from the time domain to the frequency domain [11]; that is,

$$
\sum x^{2}(t) \Delta t=\sum|X(\omega)|^{2} \Delta \omega
$$

Equation (4) reveals that the total energy of signal equals the sum of the subenergy of each frequency component. Therefore, the power entropy $S=\left\{S_{1}, S_{2}, \ldots, S_{N}\right\}$ of every natural frequency is regarded as one original signal partition; the corresponding information entropy (also called as PPSE) can be defined by

$$
E_{f}=-\sum_{i=1}^{N} q_{i} \log q_{i},
$$

where the subscript $f$ stands for the frequency domain; $q_{i}$ is the ratio of the $i$ th power spectrum to the whole spectrum, which is denoted by

$$
q_{i}=\frac{S_{i}}{\sum_{i=1}^{N} S_{i}} .
$$

Equation (5) is called the Power Spectrum Entropy (PSE) of signal $S$. When PSE is applied to fault diagnosis, the method is called PSE method. PSE explains the spectrum structure of single-channel vibration signal. The more uniformity the vibration energy distributes in the whole frequency composition, the more complex the signal is and the greater the uncertainty degree is. When the process of rotor speed-up (or -down) is constituted from multiple rotation speeds, the PSE values of different rotation speeds describe the vibration condition of whole speed-up (or -down) process. Hence, PSE method is called also Process PSE (PPSE) method.

Due to the lack of quantitative indexes for information entropy, information entropy values cannot be directly applied to diagnose rotor fault. The PPSE presenting the conditions of rotor vibration is promising to be used to distinguish rotor faults. However, the different moments and measuring points of vibration waveform always result in that PPSE values have a certain distribution range for each fault, and there is invariably some overlap among the PPSE distribution ranges of different faults [7-9]. For example, the PPSE values of four typical faults from multiple simulation experiments are listed and shown in Table 1 . Their distribution ranges have large overlap regions. When the PPSE value of an unknown fault is 5.0, it is difficult to judge to which class the unknown fault belongs. Therefore, a perfect and effective identification method needs to be proposed to address this issue. SVM method is an intelligent patter recognition method and is promising to address this overlap problem of the PPSE values of different fault modes.

\section{SVM Method}

3.1. Fundamental Theory. Support Vector Machine (SVM) [15-17] is a new machine learning method which is promising to address the realistic issues of small sample, nonlinear, and high-dimensional pattern recognition in fault diagnosis and has the advantages of complete theory, good adaptability, global optimization, short training time, and good generalization ability. SVM adopts small support vectors in training samples standing for the whole vector set to establish a SVM classifier for fault diagnosis. Assuming that the fault training sample set is $\left\{\left(x_{i}, y_{i}\right), i=1,2, \ldots, L\right\}$, where $x \in R^{n}$ and 
TABLE 1: The PPSE distribution range of rotor vibration faults.

\begin{tabular}{lc}
\hline Fault type & PSE distribution range \\
\hline Rotor imbalance & $3.659 \sim 5.260$ \\
Shaft misalignment & $3.945 \sim 5.488$ \\
Rotor rubbing & $4.357 \sim 5.618$ \\
Pedestal looseness & $3.578 \sim 5.526$ \\
\hline
\end{tabular}

$y \in|-1,+1|$, and $g(x)=(\omega \cdot x)+b$ is the general form of linear discriminated function in an $\mathrm{N}$-dimensional space, the optimal hyper plane $(\omega \cdot x)+b=0$ is able to correctly classify the data in the sample set. The distance between support vector and hyperplane is $1 /\|\omega\|$; thus, the problem of searching for hyper plane may be translated into the question of solving quadratic programming by

$$
\begin{array}{ll}
\min & \frac{1}{2}(\omega \cdot \omega)+C \sum_{i=1}^{n} \zeta_{i} \\
\text { s.t. } & y_{i}\left[\left(\omega \cdot x_{i}\right)+b\right]+\zeta_{i} \geq 1,
\end{array}
$$

where $\omega$ and $b$ are undetermined coefficients, $\zeta_{i}$ is a relaxation factor, and $C$ is a penalty factor standing for the compromise of the classification interval and error rate.

According to the optimization theory, the objective function has a unique global optimal solution because objective function and constraint conditions are convex functions. For linear programming problem, with Lagrange algorithm and $y_{i}\left[\left(w \cdot x_{i}\right)+b\right]=1$, the decision function of optimal hyperplane is

$$
f(x)=\operatorname{sgn}\left[\omega \cdot x+b^{*}\right]=\operatorname{sgn}\left(\sum_{i} a_{i} y_{i} x_{i} \cdot x+b^{*}\right) .
$$

For nonlinear programming problem, a kernel function needs to be introduced to determine the optimal hyperplane of feature vectors. The corresponding decision function is

$$
f(x)=\operatorname{sgn}\left(\sum_{i} a_{i}^{*} y_{i} k\left(x_{i}, x\right)+b^{*}\right) .
$$

The algorithm thought of optimal hyperplane in SVM is that the input vector $\mathbf{x}$ is mapped into a high-dimensional feature space $\mathbf{Z}$ by preselected nonlinear mapping to construct optimal classification hyperplane in high-dimensional feature space $\mathbf{Z}$. The output results of SVM classification function are a linear combination of intermediate nodes, each corresponding to one support vector, as shown in Figure 1.

In Figure 1, kernel functions are promising to avoid complex calculation in high-dimensional feature space, generally including linear kernel function, polynomials kernel function, RBF kernel function, and Sigmoid kernel function $[3,16-19]$. This present study selects RBF kernel function denoted by

$$
k\left(x_{i}, x\right)=\exp \left[-\frac{\left\|x-x_{i}\right\|^{2}}{\sigma^{2}}\right],
$$

where $\sigma$ is the kernel width.

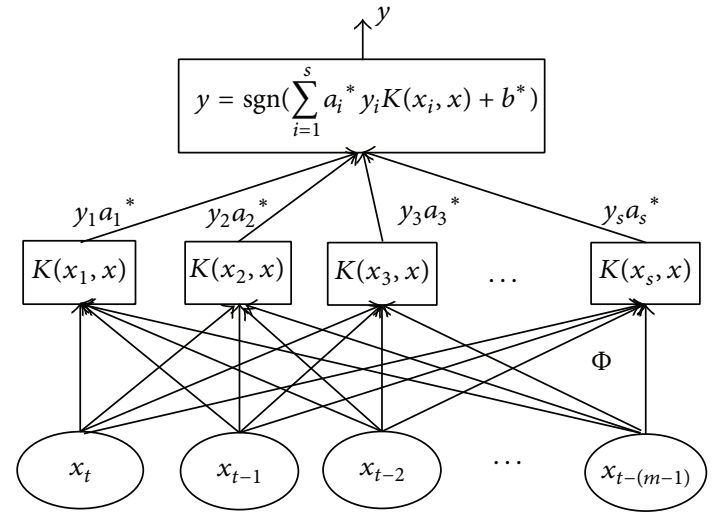

Figure 1: Support Vector Machine (SVM) schematic diagram.

3.2. SVM Multiclass Classification Method. Rotor vibration fault diagnosis is a multiclass signal process problem in practice and requires establishing a multiclass SVM classifier. The construction methods of multiclass classifier contain one-against-all (1-a-a) method, one-to-one (1-a-1) method, Directed a-Cyclic Graph SVM (DAGSVM) method, global optimization classification method, and so forth $[13,14,18]$. In this paper, the 1-a-a method was used to design SVM multiclassifier. A set of training samples is assumed as

$$
T=\left\{\left(x_{i}, y_{i}\right), \ldots,\left(x_{l}, y_{l}\right)\right\} \in(X \times Y)^{l},
$$

where $x_{i} \in X \in \mathbf{R}^{n}$ and $y_{i} \in Y=(1, \ldots, M), i=1,2, \ldots, l$.

A discrimination function $f(x)$ is searched in $\mathbf{R}^{n}$ to make each input value $x$ have one corresponding output value $y$. In fact, the essence of multiclass classification is how to find out one reasonable rule to divide all points in $\mathbf{R}^{n}$ into $M$ portions. The SVM analytical procedure of multiclass classification problem based on the 1-a-1 method is drawn as follows.

(1) Take the $j$ th class as the positive class and the rest $M-1$ classes as the negative class, according to SVM theory; the decision functions of the $j$ th class are determined by

$$
f^{j}(x)=\operatorname{sgn}\left(g^{j}(x)\right)
$$

where $g^{j}(x)=\sum_{i=1}^{l} y_{i} a_{i}^{j} K\left(x, x_{j}\right)+b^{j}, j=1, \ldots, M$.

(2) Judge the input $x$ belonging to the $j$ th class, where $j$ is the maximum label in $g^{1}(x), \ldots, g^{M}(x)$.

In light of the above steps, a multiclass classifier of samples based on SVM can be built until the surplus training samples are correctly classified.

\section{Rotor Simulation Experiment}

4.1. Select Typical Faults. Rotor imbalance, shaft misalignment, rubbing, and pedestal looseness are four typical rotor vibration faults. Rotor imbalance is often induced by unreasonable design, manufacturing and fixing error, attrition, and so forth and involves rotor mass imbalance, rotor initial bend, 
and unbalanced coupling primarily. Shaft misalignment comprises the misalignment of coupling and the misalignment of bearings. Contact rubbing constantly occurs due to the reduction of dynamic-static gap, imbalance, misalignment, and hot bend. Pedestal looseness is often caused by bad fixing and long-term vibration. In this paper, we select the four typical faults to study the rotor vibration fault diagnosis based on PPSE-SVM method.

\subsection{Simulation Experiment}

4.2.1. Test Rig. To obtain fault data, rotor test rig, as shown in Figure 2, is used to simulate four typical faults. The rotor simulation system includes test bench and measurement system. On the test bench, double rotor is opted and linked by a flexible coupling. Each rotor has one disk with equally distributed holes. Four acceleration sensors are installed on the locations A, B, C, and D of pedestal as shown in Figure 2. One speed sensor is applied to measure rotor speed. The double rotor is driven by a motor. On point $\mathrm{D}$, there is a bolt to simulate the rub-impact fault of rotor. The measurement system consists of signal acquisition instrument, signal amplifier, speed controller, velocity indicator, and computer. Signal acquisition instrument is used to collect the vibration signals of acceleration and speed sensors. In addition, due to the poor quality of sensor and measuring instrument as well as the influence of imbalance inherently, resonance and other signals maybe existent in rotor vibration signals hained from rotor experiment. However, the condition hardly influence the analytical results in this paper, because the propose method (PPSE-SVM method) is to be more effective in pure fault diagnosis of rotor vibration if the method holds acceptable diagnostic precision in the coupling fault diagnosis of rotor vibration; meanwhile, the case seems to more reasonably simulate the real rotor system.

4.2.2. Experimental Process. To study the process characteristics of rotor vibration, four kinds of typical faults (rotor imbalance, shaft misalignment, pedestal looseness, and rubbing) are simulated from $0 \mathrm{rpm}$ to $3000 \mathrm{rpm}$ on rotor vibration simulation test bench. Each fault is simulated by multiple accelerated experiments and fault data is extracted by the interval of $100 \mathrm{rpm}$ sampling speed. In measurement system, four sensors (four vibration signal channels) are fixed on rotor test rig to measure four-point vibration accelerations and these signals are gathered as original data of rotor vibration fault diagnosis. In this process, mass block is added in the holes of disk to simulate rotor imbalance fault; the shaft axes of two rotors ( $\mathrm{S} 1$ and $\mathrm{S} 2$ ) are not located on the same line to simulate the shaft misalignment of rotor system. The looseness of one (B1) or two bolts (B1 and B2) on pedestal is used to simulate the pedestal looseness fault of rotor system. The condition of bolt contacted rotor shaft is regarded to imitate rotor rubbing fault.

4.2.3. Original Data. By the simulation experiment, a mass of original vibration signals for each typical fault is gathered

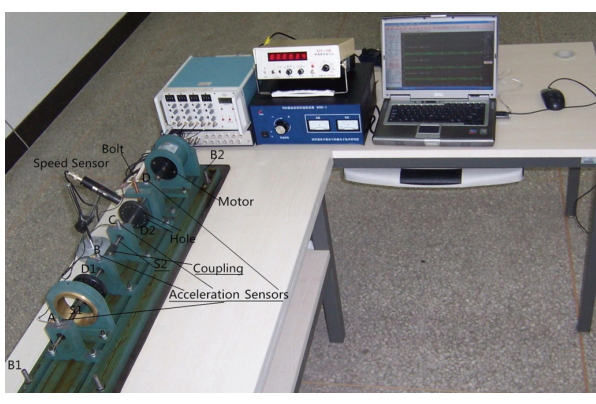

Figure 2: Rotor vibration simulation test bench and its measurement system.

when rotor speed is from 0 to $3000 \mathrm{rpm}$ with $100 \mathrm{rpm}$ sampling interval. One group of vibration signals has 30 groups of vibration waveform under one measuring point and different speeds. Therefore, in one process of speed-up or speed-down experiment, 120 groups of vibration signal waveforms for each failure modes may be collected, which reflect the process characteristics of rotor vibration faults. For point $B$, three-dimensional (3-D) frequency spectrographs of normal state and four faults are shown in Figures 3, 4, 5, 6, and 7.

4.3. Data Analysis. From Figures 3 7, the main feature of rotor fault distributes in the rotate speed range $(1000 \mathrm{rpm}$, $3000 \mathrm{rpm}$ ) with $100 \mathrm{rpm}$ sampling interval. We select the speed band to study the proposed method (PPSE-SVM method). One group of vibration signals has 21 groups of vibration waveform under one measuring point and different speeds. Therefore, in one process of speed-up or speeddown experiment, 84 groups of vibration signal waveforms for each failure modes may be collected, which reflect the process characteristics of rotor vibration faults. The speedup (or -down) process of rotor is constituted of a number of states. Vibration shapes are different under disparate rotational speeds. In fact, the vibration waveforms record the full information of rotor vibration states under different speeds and time. The vibration fault feature possesses some dispersiveness and randomness at each point; however, the vibration fault feature in rotor vibration process is regular. Information entropy matrix integrates information entropy values under multispeed and multichannel, which reflects the process regularity of vibration signals. Therefore, the information entropy matrix may be employed to describe the process regularity of rotor vibration signal. The original data which reflects the process characteristics of each fault can be gained on rotor vibration simulation experiment. According to PPSE method, the PPSE values of vibration waveform signals may be calculated. For one vibration fault, PPSE values, which fully describe the process features of this vibration fault under multichannel and multispeed, are promising to be obtained for constructing one PPSE matrix which is regarded as the fault diagnosis samples of SVM model. 


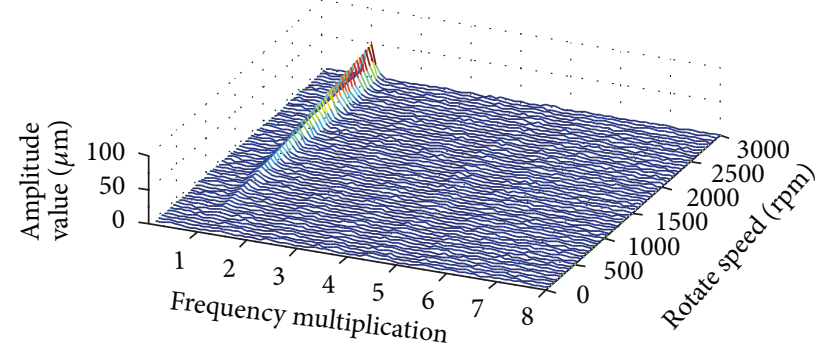

FIGURE 3: 3D frequency spectrograph of rotor normal state.

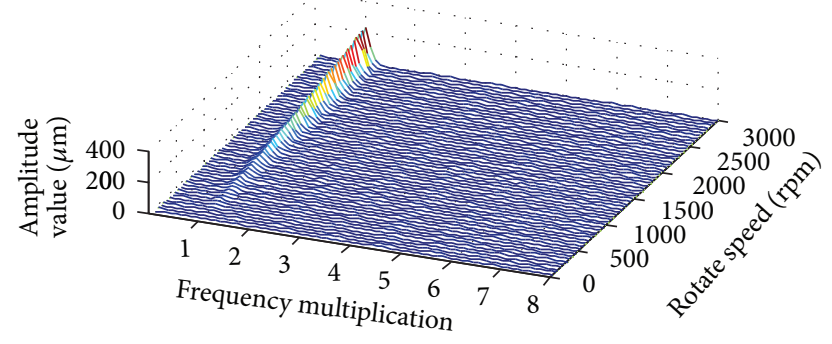

FiguRE 4: 3D frequency spectrograph of rotor imbalance.

\section{Example Analysis}

5.1. Establish PPSE-SVM Model. The basic idea of rotor vibration fault diagnosis based on PPSE-SVM method is drawn as follows: (1) gather the rotor vibration data of four typical faults based on rotor vibration fault simulation experiment; (2) extract the vibration data characteristics (PPSE values) of four faults based on PPSE method as the training and testing samples of SVM fault diagnosis model; (3) establish SVM fault diagnosis model by the training samples and accomplish the rotor vibration fault diagnosis by distinguishing fault category, discriminating failure severity, judging fault location, and validating the robustness of PPSE-SVM method. The above process is named fusion fault diagnosis based on PPSESVM method. The detailed analytical procedure is shown in Figure 8.

5.2. PPSE Feature Extraction and Parameter Selection. The PPSE values of vibration fault, which reflect the process of speed-up or speed-down, are gained based on PPSE method and Matlab simulation software. From four measuring points in the rotor simulation experiments, four groups of PPSEs are integrated to present the variation of rotor vibration condition in speed-up process. Each group of PPSE values of each channel is made up of twenty-one information entropy values which reflect the process variation of vibration signal at the corresponding measuring point during speed-up. All PPSE values of each vibration fault may constitute one PPSE matrix (or PSE matrix) which is indicated by using PPSE values, rotate speed, and measuring point (channel). The PPSE matrixes of four vibration faults are shown in Figures $9,10,11$, and 12 .

Obviously the PPSE values of each measuring point consist of sequential 21 entropy values. Hence, the 21 PPSE

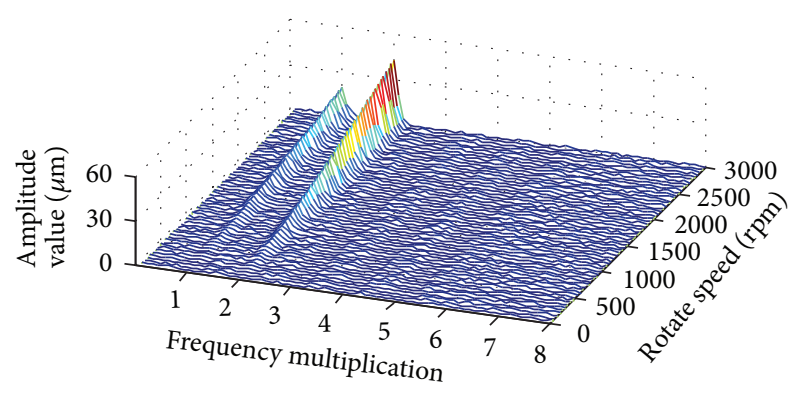

FIGURE 5: 3D frequency spectrograph of shaft misalignment.

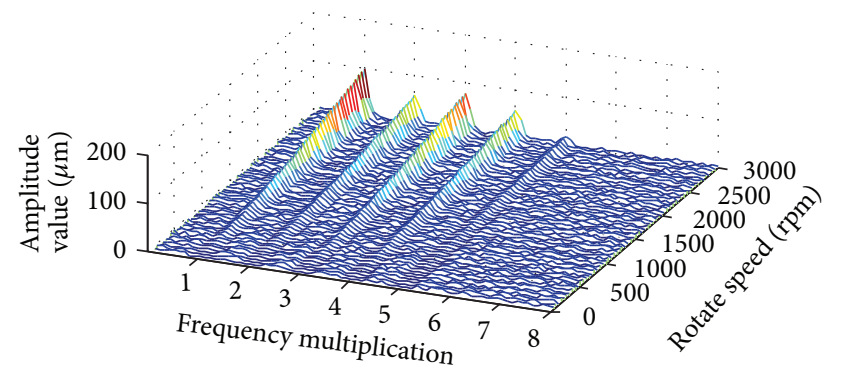

FIGURE 6: 3D frequency spectrograph of pedestal looseness.

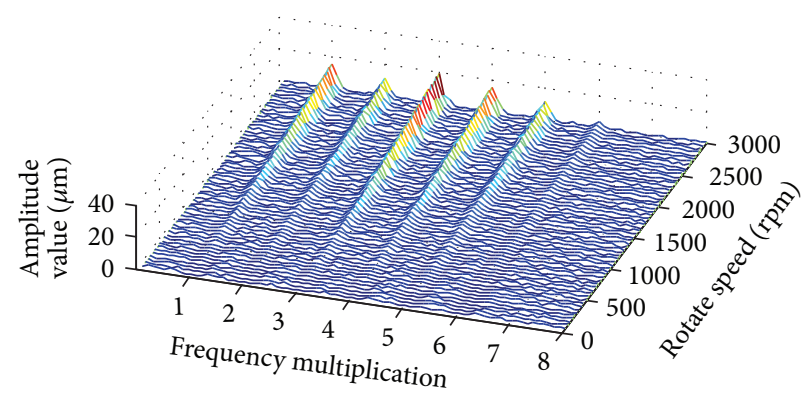

FIGURE 7: 3D frequency spectrograph of rubbing.

values were lined up as a feature vector $\left[E_{1}, E_{2}, \ldots, E_{21}\right]$, which was regarded as the fault diagnosis sample of SVM model. In light of this way, 40 fault vectors fully reflecting rotor vibration process features were taken as fault samples for each fault mode. The total number of samples of rotor vibration fault diagnosis is 160 .

SVM fault diagnosis model is structured by Matlab and Lib-SVM toolbox. The kernel function of SVM is Radial Basis Function (RBF) kernel function $\left(k\left(x_{i}, x\right)=\right.$ $\left.\exp \left[-\left\|x-x_{i}\right\|^{2} / \sigma^{2}\right]\right)$. The optimal values of $\sigma$ and $C$ are 0.07 and 136, respectively, based on random search approach [3]. Experiences show that the classification effect of $\mathrm{RBF}$ is superior to others.

5.3. Rotor Vibration Fault Category Diagnosis. The 10 fault samples of each vibration fault mode were selected as training samples to train SVM model and then obtain the optimal classifications function and establish SVM diagnosis model. Selected training samples of each fault type are shown in Table 2. 


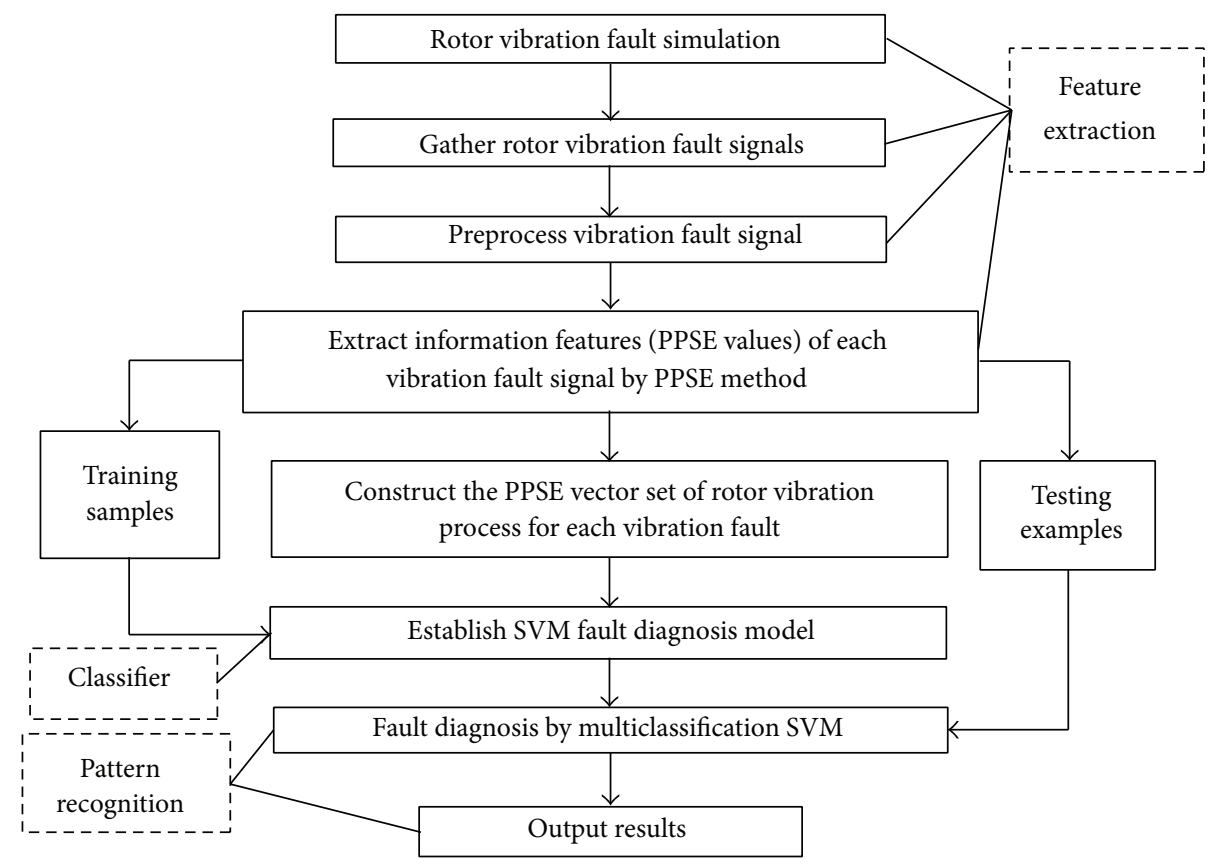

FIGURE 8: Fault diagnosis procedure for rotor vibration based on PPSE-SVM method.

TABLE 2: Partial PPSE vectors of each fault mode.

\begin{tabular}{|c|c|c|c|c|c|c|c|c|c|c|c|c|c|}
\hline Fault types & Sample number & & & & $\mathrm{PP}$ & SE vect & r $\left[E_{1}\right.$, & $E_{2}, \ldots$, & 21 & & & & Category number \\
\hline \multirow{4}{*}{ Rotor imbalance } & \multirow{2}{*}{1} & 4.315 & 4.373 & 4.466 & 4.991 & 4.864 & 4.443 & 5.258 & 5.244 & 5.131 & 5.101 & 4.576 & \multirow{2}{*}{1} \\
\hline & & 4.513 & 4.313 & 4.939 & 4.375 & 4.919 & 4.772 & 4.429 & 4.870 & 4.982 & 5.158 & & \\
\hline & \multirow{2}{*}{2} & 4.398 & 4.730 & 4.472 & 4.621 & 4.713 & 3.957 & 5.035 & 5.078 & 4.997 & 4.438 & 4.533 & \multirow{2}{*}{1} \\
\hline & & 3.731 & 4.770 & 4.539 & 3.988 & 4.802 & 4.325 & 4.553 & 5.260 & 4.080 & 3.659 & & \\
\hline \multirow{4}{*}{ Shaft misalignment } & \multirow{2}{*}{1} & 5.042 & 4.966 & 4.890 & 4.553 & 5.140 & 5.145 & 5.107 & 5.031 & 5.401 & 5.431 & 5.484 & \multirow{2}{*}{2} \\
\hline & & 5.020 & 5.126 & 5.028 & 5.191 & 4.949 & 4.817 & 4.749 & 4.503 & 4.586 & 3.981 & & \\
\hline & \multirow{2}{*}{2} & 4.790 & 4.843 & 4.750 & 4.120 & 5.327 & 5.081 & 5.288 & 5.093 & 5.338 & 5.401 & 4.944 & \multirow{2}{*}{2} \\
\hline & & 5.305 & 5.488 & 5.174 & 5.245 & 4.980 & 4.939 & 4.631 & 4.398 & 3.945 & 5.217 & & \\
\hline \multirow{4}{*}{ Rotor rubbing } & \multirow{2}{*}{1} & 5.292 & 4.867 & 4.993 & 5.185 & 5.344 & 5.081 & 5.267 & 5.463 & 5.227 & 5.469 & 5.199 & \multirow{2}{*}{3} \\
\hline & & 5.119 & 5.384 & 4.840 & 5.205 & 5.618 & 4.785 & 5.165 & 5.042 & 5.368 & 5.152 & & \\
\hline & \multirow{2}{*}{2} & 4.910 & 5.163 & 5.138 & 4.357 & 5.230 & 5.453 & 5.396 & 5.022 & 5.360 & 5.202 & 5.456 & \multirow{2}{*}{3} \\
\hline & & 5.523 & 5.570 & 5.399 & 4.720 & 3.790 & 5.061 & 5.217 & 5.053 & 5.346 & 5.137 & & \\
\hline \multirow{4}{*}{ Pedestal looseness } & \multirow{2}{*}{1} & 3.578 & 5.260 & 4.777 & 4.871 & 4.786 & 5.275 & 5.071 & 5.272 & 5.414 & 5.142 & 4.903 & \multirow{2}{*}{4} \\
\hline & & 5.315 & 5.363 & 5.311 & 5.189 & 5.019 & 5.415 & 4.358 & 5.295 & 4.175 & 5.142 & & \\
\hline & \multirow{2}{*}{2} & 4.505 & 4.908 & 5.169 & 5.366 & 5.029 & 5.361 & 5.107 & 5.286 & 5.567 & 5.038 & 5.015 & \multirow[t]{2}{*}{4} \\
\hline & & 5.316 & 5.233 & 5.339 & 5.337 & 5.502 & 5.332 & 5.290 & 5.121 & 5.529 & 5.270 & & \\
\hline
\end{tabular}

To validate the learning ability and generalization ability of SVM model, the training samples of four faults were input into the SVM diagnosis model. The result shows that these training samples are able to be completely correctly classified and the testing precision is $100 \%$. And then based on the trained SVM model, the remnant 30 samples of each vibration fault mode are classified, and the results are shown in Table 3.

From Table 3, the mean diagnostic precision of four testing samples is $96.67 \%$, which demonstrates that the trained SVM model has good learning ability and good generalization ability for rotor vibration fault classification diagnosis.

5.4. Fault Degree Diagnosis. In order to verify the validity of PPSE-SVM method in diagnosing rotor vibration fault severity, rotor pedestal looseness was selected as study object. Two failure statuses of rotor pedestal looseness (resp., one pedestal looseness (i.e., B1 looseness) and two pedestal looseness (i.e., B1 and B2 looseness)) were simulated to get fault data based on rotor test rig. The PPSE values of these data were extracted and 40 groups of data of each failure status are selected 
TABle 3: Diagnosis result of rotor vibration fault categories.

\begin{tabular}{|c|c|c|c|c|}
\hline Fault types & Testing sample number & Correct identification number & Precision & Mean precision \\
\hline Rotor imbalance & 30 & 29 & $96.67 \%$ & \multirow{4}{*}{$96.67 \%$} \\
\hline Shaft misalignment & 30 & 29 & $96.67 \%$ & \\
\hline Pedestal looseness & 30 & 28 & $93.33 \%$ & \\
\hline Rotor rubbing & 30 & 30 & $100 \%$ & \\
\hline
\end{tabular}

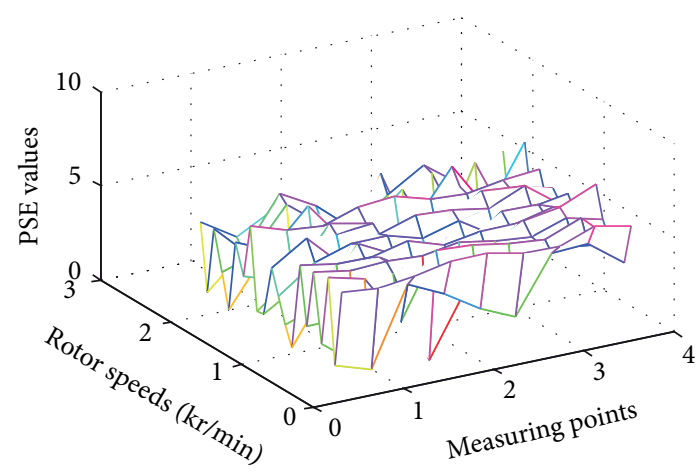

FIGURE 9: PPSE matrix of rotor imbalance fault.

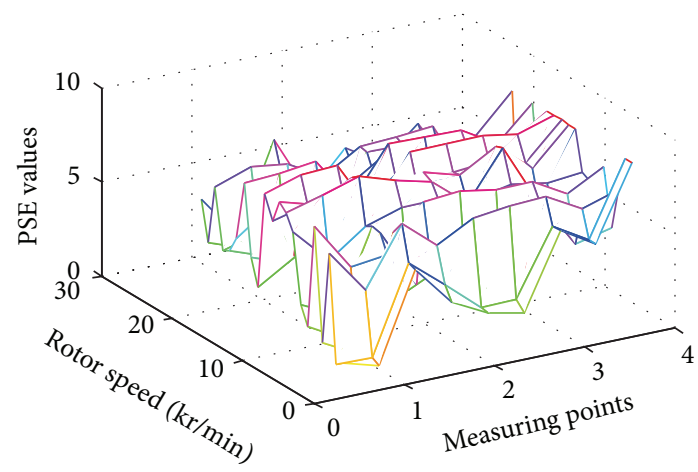

FIGURE 10: PPSE matrix of shaft misalignment fault.

as fault samples, respectively. Similarly, 10 groups of fault vectors are regarded as training samples and the surplus 30 groups are looked at as testing samples. Through training and testing SVM diagnosis model by using the training samples, the results show that the testing accuracy is also $100 \%$, which demonstrates that the trained SVM model holds good learning ability in rotor vibration fault severity diagnosis. The remaining 30 groups of testing samples of different fault degree are diagnosed by the trained SVM model. The diagnosis result of SVM model is shown in Table 4. The result illustrates that 3 samples are only mistakenly decided and the diagnosis precision is 0.95 . Therefore, PPSE-SVM method is able to availably judge rotor vibration fault degree.

5.5. Fault Point Diagnosis. Due to different measuring points corresponding to different locations on rotor vibration simulation test bench, the measuring points of sensors are selected as study object. A, B, C, and D are assumed to represent four measuring points, respectively, as shown in Figure 2. The data of measuring points are extracted as location fault data. In

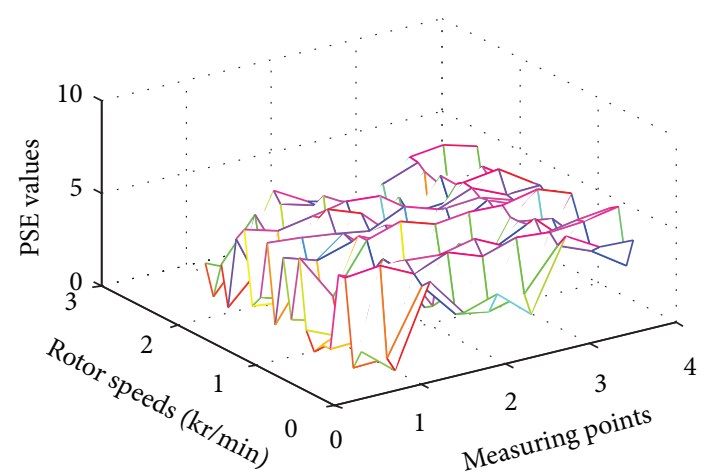

FIGURE 11: PPSE matrix of rotor rubbing fault.

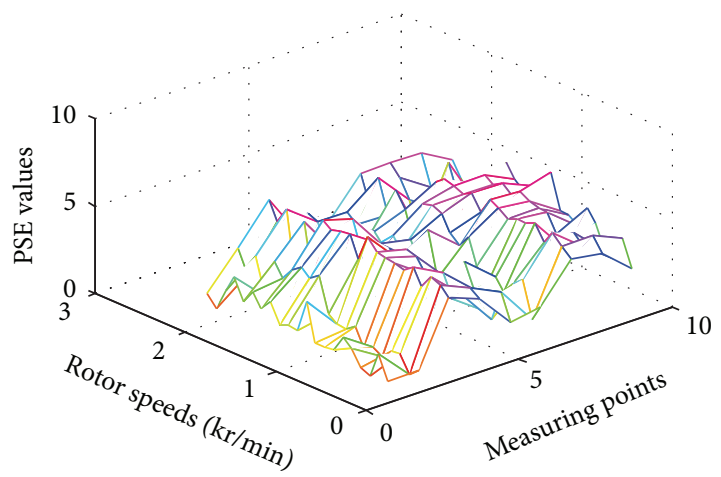

FIGURE 12: PPSE matrix of pedestal looseness fault.

a similar way, the PPSE values of these data were calculated based on PPSE-SVM method and 40 groups of fault data of each measuring point were taken as samples vectors, where 10 fault data were taken as training samples and the rest of 30 fault data were looked at as test samples for each measuring point. Through rotor vibration fault point diagnosis, the results show that the testing accuracy is $100 \%$ and the average diagnosis precision is $93.33 \%$, which is shown in Table 5. It is indicated that the PPSE-SVM diagnosis method is feasible and effective in rotor vibration fault point diagnosis.

5.6. Robustness Verification. To validate the robustness of PPSE-SVM method in rotor vibration fault diagnoses, including judgments of fault category, severity, and location, the original signals of the aforementioned test samples of three fault types were overlapped by Gaussian white noise with mean value 0 and variance 5. And then the PPSE values of these original signals of vibration fault were gained as new test samples based on PPSE method. Ultimately these new test samples of three fault types were inputted in 
TABLE 4: Diagnosis results of fault severity.

\begin{tabular}{lcccc}
\hline Fault degree & Test sample number & Correct identification number & Precision & Mean precision \\
\hline One pedestal looseness & 30 & 29 & $96.67 \%$ & $95 \%$ \\
Two pedestal looseness & 30 & 28 & $93.33 \%$ & \\
\hline
\end{tabular}

TABLE 5: Diagnosis results of rotor vibration fault locations.

\begin{tabular}{|c|c|c|c|c|}
\hline Fault points & Test sample number & Correct identification number & Precision & Mean precision \\
\hline A & 30 & 28 & $93.33 \%$ & \multirow{4}{*}{$93.33 \%$} \\
\hline B & 30 & 29 & $96.67 \%$ & \\
\hline $\mathrm{C}$ & 30 & 28 & $93.33 \%$ & \\
\hline $\mathrm{D}$ & 30 & 27 & $90 \%$ & \\
\hline
\end{tabular}

TABLE 6: Verification results of the robustness of PPSE-SVM method in rotor vibration fault category, severity, and locations.

\begin{tabular}{|c|c|c|c|c|c|}
\hline Fault type & Test sample number & Correct number & Precision & Reduction precision & Mean precision \\
\hline Category & 120 & 115 & $95.83 \%$ & $0.83 \%$ & \multirow{3}{*}{$94.33 \%$} \\
\hline Severity & 60 & 56 & $93.33 \%$ & $1.67 \%$ & \\
\hline Location & 120 & 112 & $93.33 \%$ & 0 & \\
\hline
\end{tabular}

the corresponding SVM fault diagnosis models trained in Sections 5.3 5.5. The analysis results are shown in Table 6.

As shown in Table 6, after overlapping Gaussian noise on the original vibration signals of test samples, the diagnosis precisions of three fault types (category, severity, and location) are $0.9583,0.9333$, and 0.9333 , respectively, and only reduce by $0.0083,0.0167$, and 0 relative to those before superposition severally, so that the mean precision of rotor vibration fault diagnosis comes to 0.9433 . The results reveal that the proposed PPSE-SVM method has good fault-tolerant capability and strong robustness in antinoise interference.

\section{Conclusion}

The objective of these efforts is attempted to advance a process fault diagnosis method-Process Power Spectrum Entropy and Support Vector Machine (PPSE-SVM) method-by fusing the advantages of information entropy method and SVM theory for rotor vibration fault diagnosis from a process perspective based on information fusion technique. Through rotor vibration fault diagnosis based on PPSE-SVM method, some conclusions are drawn as follows.

(1) The Process Power Spectrum Entropy (PPSE) values can effectively reflect the process variation of rotor vibration signals.

(2) PPSE-SVM model can be established by small samples, PPSE feature vectors extracted from the fault vibration data of rotor fault vibration simulation experiments.

(3) The PPSE-SVM model trained by PPSE feature vectors is demonstrated to be an efficient fault diagnosis model, which possesses strong learning ability, generalization ability, and fault tolerance ability because of high testing precision (100\%), high diagnosis precision (resp., 0.9667, 0.95, and 0.9333), and strong antinoise interference ability (0.9433 in precision) in the rotor vibration fault diagnosis and analysis on fault category, failure severity, fault points, and robustness from a process perspective.

(4) The presented PPSE-SVM method is also proved to be effective and reasonable, and this study provides a promising diagnosis technology for rotor vibration fault.

(5) Some idealized factors were considered for rotor vibration fault diagnosis based on the PPSE-SVM method on rotor vibration simulation test bench in this paper. For complex machinery, like an aeroengine, the validity of the presented PPSE-SVM method needs to be further verified in vibration fault diagnosis.

\section{Conflict of Interests}

The authors declare that they have no conflict of interests regarding the publication of this paper.

\section{Acknowledgments}

This paper is supported by the National Natural Science Foundation of China (Grant nos. 51175017 and 51275024) and Research Foundation for the Doctoral Program of Higher Education (no. 2011112110011). The authors would like to thank them.

\section{References}

[1] D. J. Ewins, "Control of vibration and resonance in aero engines and rotating machinery-an overview," International Journal of Pressure Vessels and Piping, vol. 87, no. 9, pp. 504-510, 2010. 
[2] F. Al-Badour, M. Sunar, and L. Cheded, "Vibration analysis of rotating machinery using time-frequency analysis and wavelet techniques," Mechanical Systems and Signal Processing, vol. 25, no. 6, pp. 2083-2101, 2011.

[3] C. W. Fei and G. C. Bai, "Wavelet correlation feature scale entropy and fuzzy support vector machine approach for aeroengine whole-body vibration fault diagnosis," Shock and Vibration, vol. 20, no. 2, pp. 341-349, 2013.

[4] P. W. Tse, S. Gontarz, and X. J. Wang, "Enhanced eigenvector algorithm for recovering multiple sources of vibration signals in machine fault diagnosis," Mechanical Systems and Signal Processing, vol. 21, no. 7, pp. 2794-2813, 2007.

[5] J.-D. Wu and C.-H. Liu, "An expert system for fault diagnosis in internal combustion engines using wavelet packet transform and neural network," Expert Systems with Applications, vol. 36, no. 3, pp. 4278-4286, 2009.

[6] L. A. Overbey and M. D. Todd, "Dynamic system change detection using a modification of the transfer entropy," Journal of Sound and Vibration, vol. 322, no. 1-2, pp. 438-453, 2009.

[7] L. Qu, L. Li, and J. Lee, "Enhanced diagnostic certainty using information entropy theory," Advanced Engineering Informatics, vol. 17, no. 3-4, pp. 141-150, 2003.

[8] H. Cui, L. Zhang, R. Kang, and X. Lan, "Research on fault diagnosis for reciprocating compressor valve using information entropy and SVM method," Journal of Loss Prevention in the Process Industries, vol. 22, no. 6, pp. 864-867, 2009.

[9] X. Xing, "Physical entropy, information entropy and their evolution equations," Science in China A: Mathematics, Physics, Astronomy, vol. 44, no. 10, pp. 1331-1339, 2001.

[10] H. Endo and R. B. Randall, "Enhancement of autoregressive model based gear tooth fault detection technique by the use of minimum entropy deconvolution filter," Mechanical Systems and Signal Processing, vol. 21, no. 2, pp. 906-919, 2007.

[11] J. Wang, Y. T. Ai, X. F. Liu et al., "Study on quantitative diagnosis method of rotor vibration faults based on process information entropy," in Proceedings of the 2nd International Conference on Computer Engineering and Technology (ICCET '10), pp. V5-407V5-411, Chengdu, China, April 2010.

[12] J. Ye, "Fault diagnosis of turbine based on fuzzy cross entropy of vague sets," Expert Systems with Applications, vol. 36, no. 4, pp. 8103-8106, 2009.

[13] N. García-Pedrajas and D. Ortiz-Boyer, "An empirical study of binary classifier fusion methods for multiclass classification," Information Fusion, vol. 12, no. 2, pp. 111-130, 2011.

[14] W. Ding and J. Yuan, "Spike sorting based on multi-class support vector machine with superposition resolution," Medical and Biological Engineering and Computing, vol. 46, no. 2, pp. 139-145, 2008.

[15] Z.-W. Guo and G.-C. Bai, "Classification using least squares support vector machine for reliability analysis," Applied Mathematics and Mechanics, vol. 30, no. 7, pp. 853-864, 2009.

[16] A. Widodo and B.-S. Yang, "Support vector machine in machine condition monitoring and fault diagnosis," Mechanical Systems and Signal Processing, vol. 21, no. 6, pp. 2560-2574, 2007.

[17] S.-F. Yuan and F.-L. Chu, "Support vector machines-based fault diagnosis for turbo-pump rotor," Mechanical Systems and Signal Processing, vol. 20, no. 4, pp. 939-952, 2006.

[18] M. Saimurugan, K. I. Ramachandran, V. Sugumaran, and N. R. Sakthivel, "Multi component fault diagnosis of rotational mechanical system based on decision tree and support vector machine," Expert Systems with Applications, vol. 38, no. 4, pp. 3819-3826, 2011.
[19] W. T. Peter, Y. H. Peng, and R. Yam, "Wavelet analysis and envelop detection for rolling element bearing fault diagnosis their effectives and flexibilities," Journal of Vibration and Acoustics, vol. 12, no. 3, pp. 303-310, 2000. 

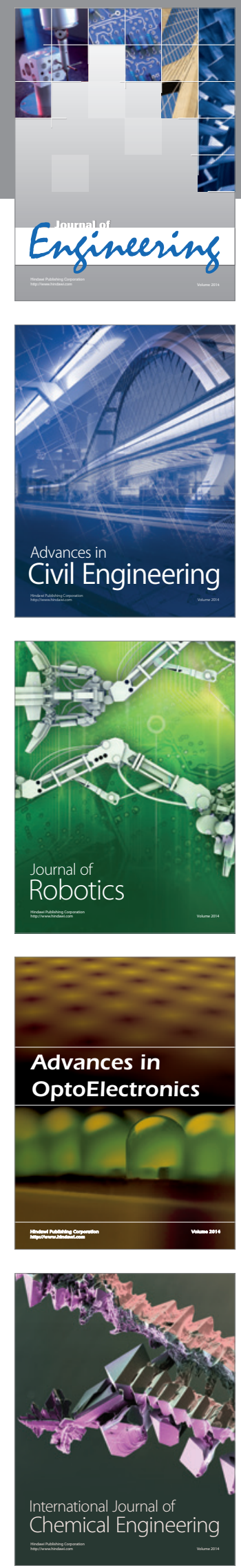

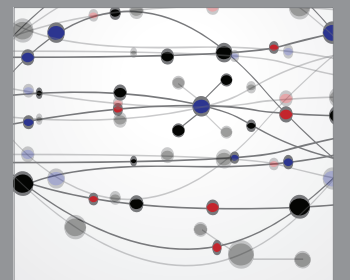

The Scientific World Journal
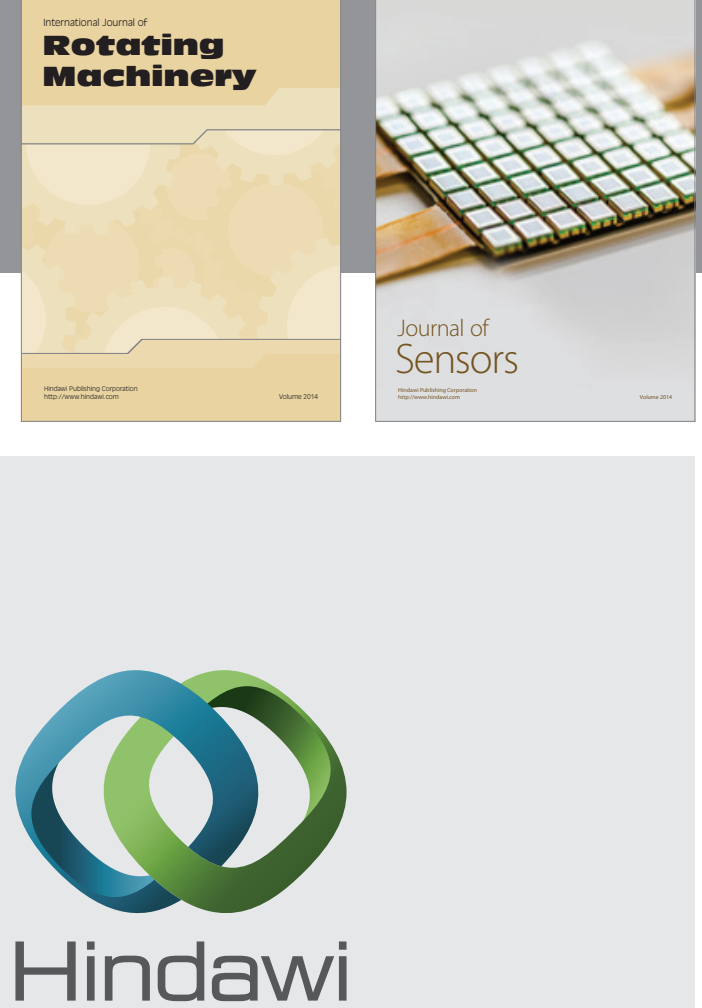

Submit your manuscripts at http://www.hindawi.com

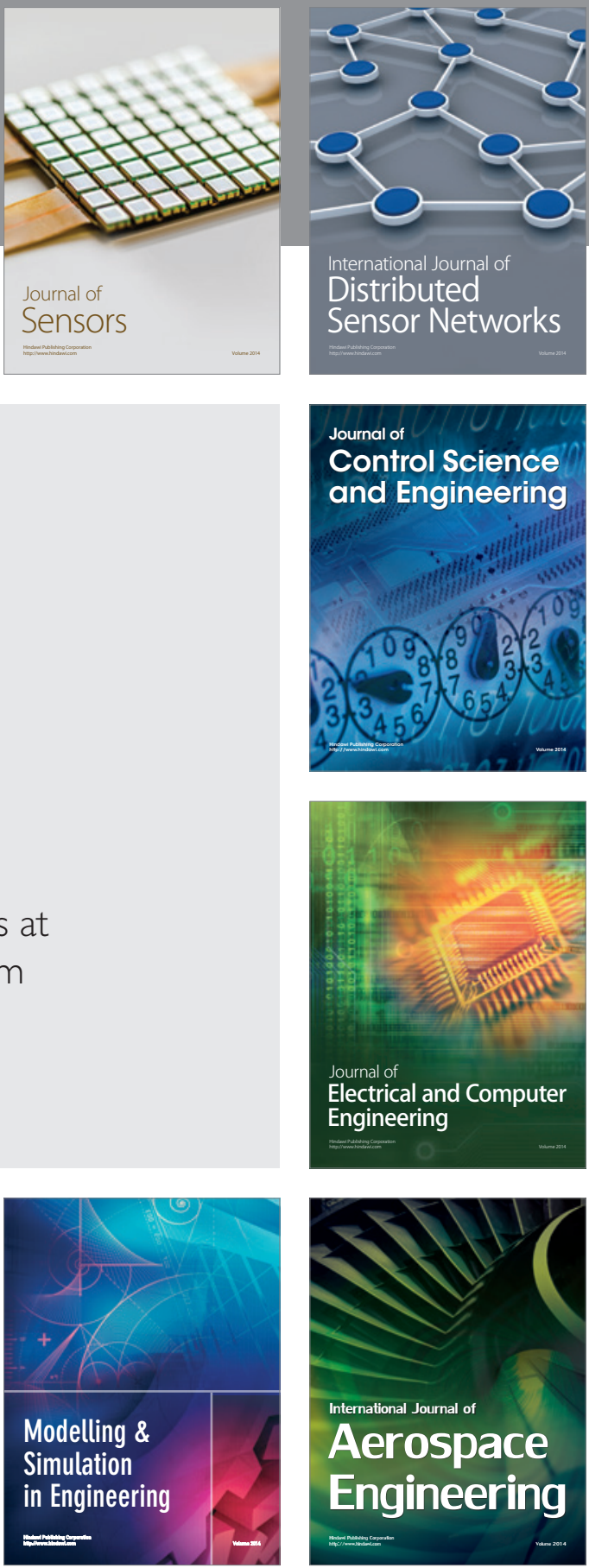

Journal of

Control Science

and Engineering
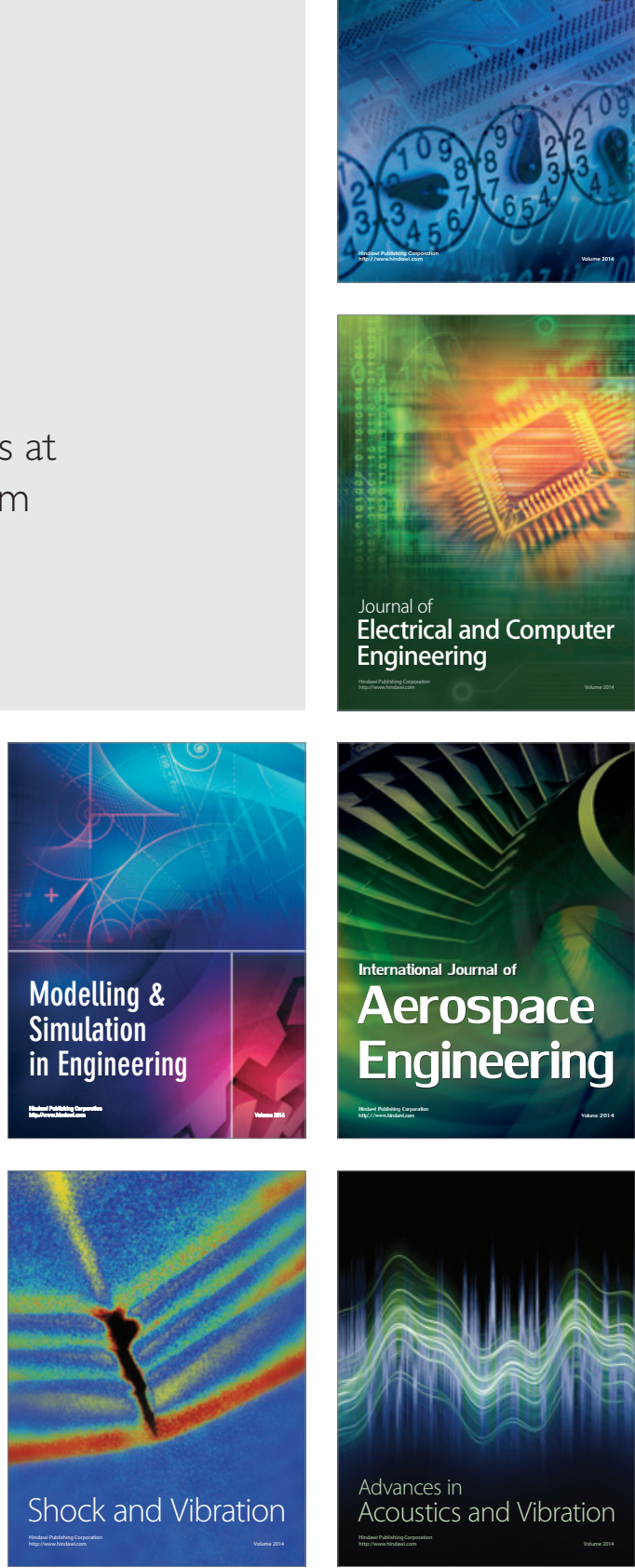